\title{
The minimum effort required to eradicate infections in models with backward bifurcation
}

\author{
Muntaser Safan • Hans Heesterbeek • \\ Klaus Dietz
}

Received: 10 June 2005 / Revised: 28 April 2006 /

Published online: 5 August 2006

(C) Springer-Verlag 2006

\begin{abstract}
We study an epidemiological model which assumes that the susceptibility after a primary infection is $r$ times the susceptibility before a primary infection. For $r=0(r=1)$ this is the SIR (SIS) model. For $r>1+(\mu / \alpha)$ this model shows backward bifurcations, where $\mu$ is the death rate and $\alpha$ is the recovery rate. We show for the first time that for such models we can give an expression for the minimum effort required to eradicate the infection if we concentrate on control measures affecting the transmission rate constant $\beta$. This eradication effort is explicitly expressed in terms of $\alpha, r$, and $\mu$. As in models without backward bifurcation it can be interpreted as a reproduction number, but not necessarily as the basic reproduction number. We define the relevant reproduction numbers for this purpose. The eradication effort can be estimated from the endemic steady state. The classical basic reproduction number $R_{0}$ is smaller than the eradication effort for $r>1+(\mu / \alpha)$ and equal to the effort for other values of $r$. The method we present is relevant to the whole class of compartmental models with backward bifurcation.
\end{abstract}

Dedicated to Karl Peter Hadeler on the occasion of his 70th birthday.

M. Safan $(\bowtie) \cdot$ K. Dietz

Department of Medical Biometry Eberhard-Karls-University Tübingen, Westbahnhofstr. 55, 72070, Tübingen, Germany

e-mail:msafan@mans.edu.eg; safan.muntaser@uni-tuebingen.de

M. Safan

Mathematics Department, Faculty of Science, Mansoura University, 35516 Mansoura, Egypt

J. A. P. Heesterbeek

Faculty of Veterinary Medicine, University of Utrecht, Yalelaan 7,

3584 CL Utrecht, The Netherlands 
Keywords Epidemic model - Backward bifurcation - Reproduction numbers . Eradication effort

\section{Introduction}

The simultaneous presence of multiple endemic steady states (i.e. the backward bifurcation phenomenon) has in recent years been the topic of many papers on infectious disease models (e.g. [1-3, 6-8,10,12-15,13-16]). We aim in this paper to develop a method to estimate the effort needed to eradicate an infectious agent, starting from an endemic steady state, in systems that allow backward bifurcation.

We develop our method using a special case of a model proposed by Hadeler and Castillo-Chavez [7]. In this model individuals can return to the susceptible state after each infection. We distinguish susceptibles that have never experienced the infection (naive individuals) from susceptibles that have a prior infection history (independent of the number of repeated infections). Hadeler and Castillo-Chavez showed that backward bifurcation can occur in their model. Our model defines a whole family of infectious disease models which contains on one end the SIR model (i.e. individuals have zero susceptibility after the first and only infection, i.e. they are fully immune). The key parameter of our model is the ratio $r$ of the susceptibility after a primary infection and the susceptibility before a primary infection. For the SIR model, $r=0$. For the SIS model we have $r=1$. We shall provide a lower bound of $r$ for which backward bifurcations are possible. When they occur, then $r>1$, i.e. after a primary infection the susceptibility is higher than before. This could be interpreted as immuno-suppression, which may be relevant for some infections like pertussis where backward bifurcations have been studied in a slightly more complicated model by van Boven et al. [2], or as sensitization to new infections after having experienced a primary infection. We describe the model and its steady states in Sect. 2. In Sect. 3 we determine the minimum eradication effort and show how this can be obtained from the stable endemic equilibrium. Since for models without backward bifurcation the minimum eradication effort is identical to the basic reproduction number $R_{0}$ [5], we investigate to what extent this result still holds true for this model with backward bifurcation (Sect. 4). It turns out that it is the interplay between two new reproduction numbers that determines the outcome. Both these new reproduction numbers coincide with $R_{0}$ for the model without backward bifurcation. In Sect. 5 we shall determine for which initial conditions the minimum eradication effort can be interpreted in terms of these new reproduction numbers. Finally in Sect. 6 we discuss our results.

\section{The model and its equilibria}

Consider a demographically stationary population structured into three classes of individuals according to their epidemiological status. The first class is that of susceptibles without past infections $S_{0}(t)$, i.e. individuals who never were 
infected and may contract the infection (to be referred to as naive individuals); the second class is that of infectious individuals $I(t)$; and the third class is that of susceptibles with at least one past infection $S_{1}(t)$, to be referred to as recovered. We model in terms of fractions and assume that the total size is equal to one, $S_{0}+I+S_{1}=1$.

The states $S_{0}, S_{1}$, and $I$ can in principle be distinguished from each other by performing simultaneously two tests: the first measures the level of antibodies $(\mathrm{AB})$, i.e. the presence of immunological memory and the other is a test for the presence of the infective agent (such as a PCR test). If the AB and PCR test results for a given individual are both negative, we classify the individual in question naive, i.e. with current state $S_{0}$. If either test gives a positive result we classify the individual as having state $S_{1}$ (when AB is positive, but PCR is not) or state $I$ (when PCR is positive, regardless of the AB result). This applies to all infections with antibody-mediated immunity for which sensitive $\mathrm{AB}$ and PCR test are available.

Assume that individuals are born naive with per capita birth rate $\mu$. Naive individuals can either die with per capita death rate $\mu$ or they get infected with linear incidence rate $\beta I=r_{0} \kappa I$ where $\kappa$ is the per capita contact rate and $r_{0}$ is the probability of success of contacts between infected and naive individuals and hence, $\beta$ is the successful contact rate between $S_{0}$ and $I$. Infected individuals can either die with per capita death rate $\mu$ or recover with per capita rate $\alpha$. Recovered individuals can either die with per capita death rate $\mu$ or get infected again with linear incidence rate $\tilde{\beta} I=r_{1} \kappa I$, where $r_{1}$ is the probability of success of contacts between $I$ and $S_{1}$ and hence, $\tilde{\beta}$ is the successful contact rate between infected and recovered individuals. Define $r=\left(r_{1} / r_{0}\right)$ as the ratio of transmission probabilities. Hence, the successful contact rate between infecteds and recovereds is $r \beta$. These assumptions lead to the following system of ordinary differential equations:

$$
\begin{aligned}
\frac{\mathrm{d} S_{0}}{\mathrm{~d} t} & =\mu-(\mu+\beta I) S_{0}, \\
\frac{\mathrm{d} I}{\mathrm{~d} t} & =\beta\left(S_{0}+r S_{1}\right) I-(\alpha+\mu) I, \\
\frac{\mathrm{d} S_{1}}{\mathrm{~d} t} & =\alpha I-(\mu+r \beta I) S_{1} .
\end{aligned}
$$

When $r=0$, the model is an SIR model; when $r=1$, the model becomes an SIS model. For system (1) we have

$$
R_{0}=\frac{\beta}{\alpha+\mu} .
$$

Note that $r$ plays no role in $R_{0}$ since in the invasion phase in a homogeneously mixing population, the probability that a recovered individual comes into contact again with an infectious individual is neglected. 
For both extreme cases $r=0$ (SIR) and $r=1$ (SIS), it is well-known [4] that the infection-free equilibrium (IFE) is globally stable for $R_{0} \leq 1$, and that there exists a globally stable endemic state for $R_{0}>1$.

\subsection{Forward bifurcations}

If $0<r<1+(\mu / \alpha)$, then the dynamical system (1) has the following endemic states:

(1) The infection-free equilibrium $(1,0,0)$, which is globally asymptotically stable if and only if $R_{0} \leq 1$.

(2) The endemic equilibrium $\left(\bar{S}_{0}, \bar{I}, \bar{S}_{1}\right)$, which is unique and is globally asymptotically stable if and only if $R_{0}>1$, where:

$$
\begin{aligned}
\bar{I}= & \frac{1}{2}\left(\left(1-\frac{1}{r R_{0}}-\frac{\mu}{(\alpha+\mu) R_{0}}\right)\right. \\
& +\sqrt{\left.\left(1-\frac{1}{r R_{0}}-\frac{\mu}{(\alpha+\mu) R_{0}}\right)^{2}+\frac{4 \mu\left(1-\frac{1}{R_{0}}\right)}{(\alpha+\mu) r R_{0}}\right),} \\
\bar{S}_{0}= & \frac{\mu}{\mu+\beta \bar{I}}, \\
\bar{S}_{1}= & \frac{\alpha \bar{I}}{\mu+r \beta \bar{I}} .
\end{aligned}
$$

For $R_{0}>1 / r$ and $\alpha \gg \mu$, the endemic equilibrium for $I$ is approximately equal to $1-\left(1 / r R_{0}\right)$. Although the endemic level $\bar{I}$ increases monotonically with $R_{0}$, there are two inflection points close to $R_{0}=1 / r$. Both the point with the minimal and the maximal slope are close to each other (Fig. 1a).

\subsection{Backward bifurcation model}

For $r>1+(\mu / \alpha)$, the model (1) exhibits a backward bifurcation. Apart from the infection-free equilibrium $\left(S_{0}, I, S_{1}\right)=(1,0,0)$ there can exist a single unique endemic steady state or two positive steady states depending on the solutions to a quadratic equation. The endemic steady state values for $S_{0}$ and $S_{1}$ are as given above in (2), where, $\bar{I} \in[0,1]$ is the solution of the quadratic equation:

$$
f(I)=r \beta^{2} I^{2}+(r \mu-(r \beta-(\alpha+\mu))) \beta I+\mu(\alpha+\mu-\beta)=0 .
$$

This equation has one or two feasible (i.e. positive, real) solutions, depending on the values of the parameters. As seen in the previous subsection, there is one solution, given in (2), for the case when $R_{0}>1$ and arbitrary $r>0$. For $R_{0}<1$ the situation is more complicated. For $r>1+(\mu / \alpha)$ there is a region of values for $R_{0}<1$ where there are two feasible solutions: 

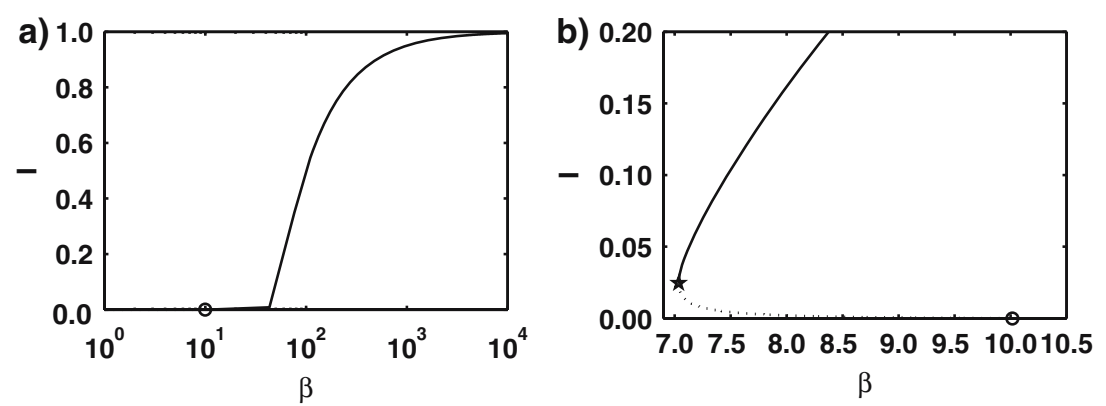

Fig. 1 This figure shows the endemic prevalence $I$ as a function of the contact rate $\beta$ for two values of $r(r=0.2$ part (a), $r=1.5$ part (b), $\alpha=10$ per year and $\mu=0.015$ per year). For $r$ between 0 and $1+\mu / \alpha$, there are two inflection points close to $R_{0}=1 / r$. For $R_{0}>1 / r$, the endemic equilibrium is close to $1-1 / r R_{0}(a)$. For $r>1+\mu / \alpha$, there are three equilibrium points between $R_{0}=R_{0}^{\star}$ and $R_{0}=1$ (b)

$$
\begin{aligned}
\bar{I}^{ \pm}= & \frac{1}{2}\left(\left(1-\frac{1}{r R_{0}}-\frac{\mu}{(\alpha+\mu) R_{0}}\right)\right. \\
& \pm \sqrt{\left.\left(1-\frac{1}{r R_{0}}-\frac{\mu}{(\alpha+\mu) R_{0}}\right)^{2}+\frac{4 \mu\left(1-\frac{1}{R_{0}}\right)}{(\alpha+\mu) r R_{0}}\right)} .
\end{aligned}
$$

\subsection{The critical contact rate $\beta^{\star}$}

We denote the two endemic steady states by $E^{+}$and $E^{-}$. We now refer to Fig. $1 \mathrm{~b}$ for additional notation for the situation $r>1+(\mu / \alpha)$. On the vertical axis we give the $I$-component of $E^{+}$and $E^{-}$. On the horizontal axis we vary the contact rate $\beta$. In other words, on the horizontal axis we vary $R_{0}$, but we assume that all ingredients of $R_{0}$ other than $\beta$ are constant. When moving from $R_{0}>1$ to $R_{0}<1$ we only decrease $\beta$. The effect is that the corresponding values for the $I$-component of $E^{+}$and $E^{-}$will come closer together. The steady states coincide in the turning point [18] $E^{\star}$, with $I$-component $I^{+}=I^{-}=: I^{\star}$. The value of $\beta$ and $R_{0}$ for which that happens will be denoted by $\beta^{\star}$ and $R_{0}^{\star}$, respectively. It is a straightforward calculation from (4) to obtain that at the turning point

$$
\begin{aligned}
\beta^{\star} & =\frac{(\sqrt{\mu(r-1)}+\sqrt{\alpha})^{2}}{r} \text { for } r \geq 1+\frac{\mu}{\alpha}, \\
R_{0}^{\star} & =\frac{\beta^{\star}}{\alpha+\mu}, \\
I^{\star} & =\frac{r \beta^{\star}-r \mu-(\alpha+\mu)}{2 r \beta^{\star}} .
\end{aligned}
$$

For $r \leq 1+\mu / \alpha$ we have $\beta^{\star}=\alpha+\mu$. 


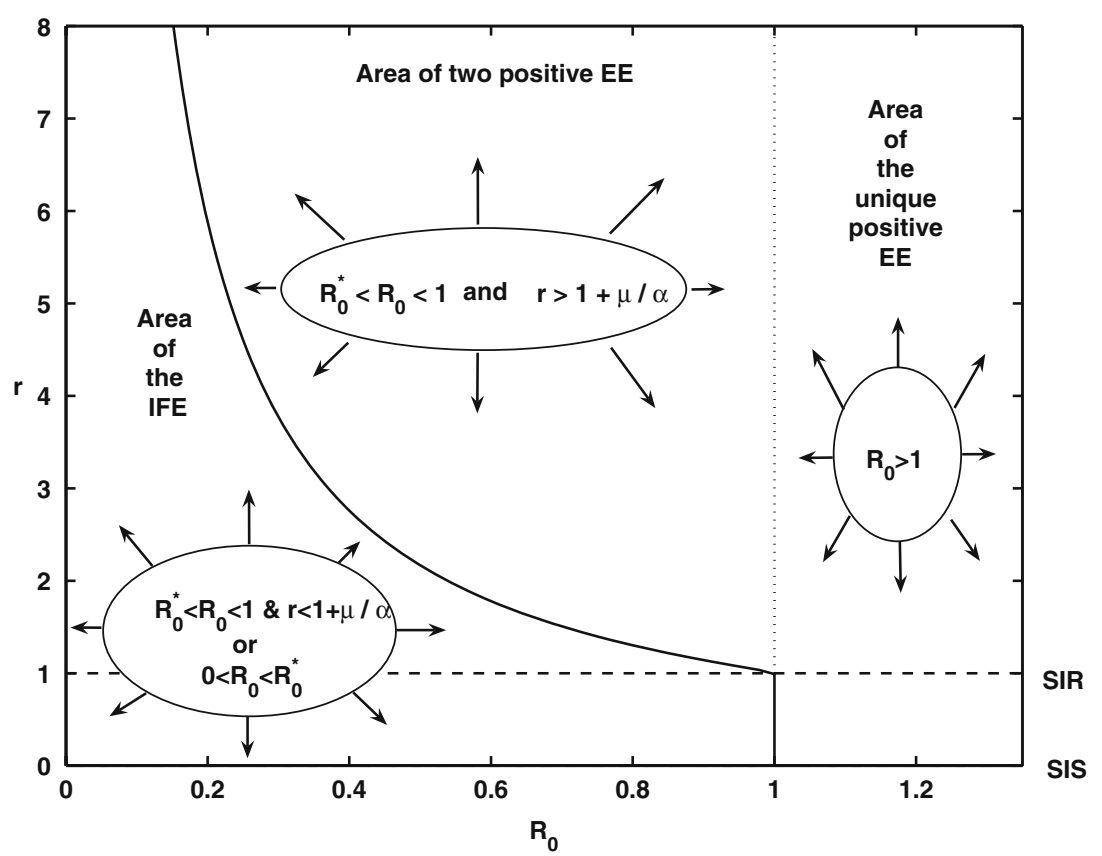

Fig. 2 Bifurcation diagram in the $\left(R_{0}, r\right)$ plane showing the partition of the plane according to the number of the endemic equilibrium points ( $\alpha=10$ per year and $\mu=0.015$ per year)

The following proposition summarizes the behaviour. Figure 2 shows the partition of the $\left(R_{0}, r\right)$-plane with the same information about the steady states.

\section{Proposition For:}

(a) $R_{0}>1$, a unique positive endemic equilibrium exists in addition to the infection-free equilibrium,

(b) $R_{0}^{\star}<R_{0}<1$ and $r>1+\mu / \alpha$ two positive endemic equilibria exist in addition to the IFE,

(c) $0<R_{0}<R_{0}^{\star}$ or $\left(R_{0}^{\star}<R_{0}<1\right.$ and $\left.r<1+\mu / \alpha\right)$, there is only the IFE.

Proof From (3), one can simply evaluate:

$$
\begin{aligned}
f(1) & =\beta(r \mu+\alpha)+\mu(\alpha+\mu), \quad f(0)=\mu(\alpha+\mu)\left(1-R_{0}\right), \\
f^{\prime}(0) & =\beta(r \mu+\alpha+\mu-r \beta) .
\end{aligned}
$$

It is easy to check that $f^{\prime}\left(I^{\star}\right)=0$ where $I^{\star}=\frac{r \beta-r \mu-(\alpha+\mu)}{2 r \beta}>0$ iff $\beta>\mu+$ $(\alpha+\mu) / r$.

It is clear that $f(1)>0$, that $f(0)$ can be positive or negative depending on the value of the basic reproduction number $R_{0}$, and that $f^{\prime}(0)$ can be positive or negative depending on the values of the parameters. If $f(0)>0, f^{\prime}(0)<0$ 
and $f\left(I^{\star}\right)<0$, there are two positive endemic equilibria in addition to the infection-free equilibrium. If $f(0)<0$ and $f^{\prime}(0)>0$, then there is a unique positive endemic state in addition to the infection-free equilibrium. Otherwise there is only the infection-free equilibrium. It is easy to check that: $f(0)>0$ if and only if $R_{0}<1, f^{\prime}(0)<0$ if and only if $R_{0}>(\mu /(\alpha+\mu))+(1 / r), f\left(I^{\star}\right)<0$ if and only if $R_{0}>R_{0}^{\star}$. Combining the conditions together finishes the proof.

For $r>1+(\mu / \alpha)$, we have indicated the turning point where the two positive endemic states merge by $E^{\star}=\left(S_{0}^{\star}, I^{\star}, S_{1}^{\star}\right)$ where

$$
\begin{aligned}
& S_{0}^{\star}=\frac{1}{1+\sqrt{\frac{\alpha}{\mu(r-1)}}}\left(\frac{r}{r-1}\right), \\
& I^{\star}=\frac{1}{1+\sqrt{\frac{\alpha}{\mu(r-1)}}}\left(1-S_{0}^{\star}\right), \\
& S_{1}^{\star}=\frac{\sqrt{\frac{\alpha}{\mu(r-1)}}}{1+\sqrt{\frac{\alpha}{\mu(r-1)}}}\left(1-S_{0}^{\star}\right] .
\end{aligned}
$$

\section{The minimum effort $\mathcal{R}$ required to eradicate an infection}

In the last section, we saw that a reduction of the contact rate $\beta$ below its critical value $\beta^{\star}$ would reduce the equilibrium prevalence to zero, for the region of values for $r$ where our system exhibits a backward bifurcation. For the region $r<1+\mu / \alpha$ we found that $\beta^{\star}=\alpha+\mu$, so also there $\beta^{\star}$ is the value of $\beta$ separating between the existence of the zero and endemic steady state. Suppose we concentrate our control effort on reducing contacts. Given this one could define a measure for the control effort required to eradicate an infection in such a system when starting from a situation where the contact rate has the value $\beta$. Can we find a measure that indicates minimum effort needed, starting from an endemic state, and express this in terms of measurable steady state fractions? We propose the following simple quantity

$$
\mathcal{R}:=\frac{\beta}{\beta^{\star}} .
$$

We will interpret this quantity as a measure of eradication effort. For the SIR and SIS case, and more generally for $r \leq 1+(\mu / \alpha)$, we have (Sect. 2) that $\beta^{\star}=\alpha+\mu$ and this gives $\mathcal{R}=R_{0}$ and we see that such an interpretation is justified. For the interpretation for arbitrary $r$ we first show the following proposition. 


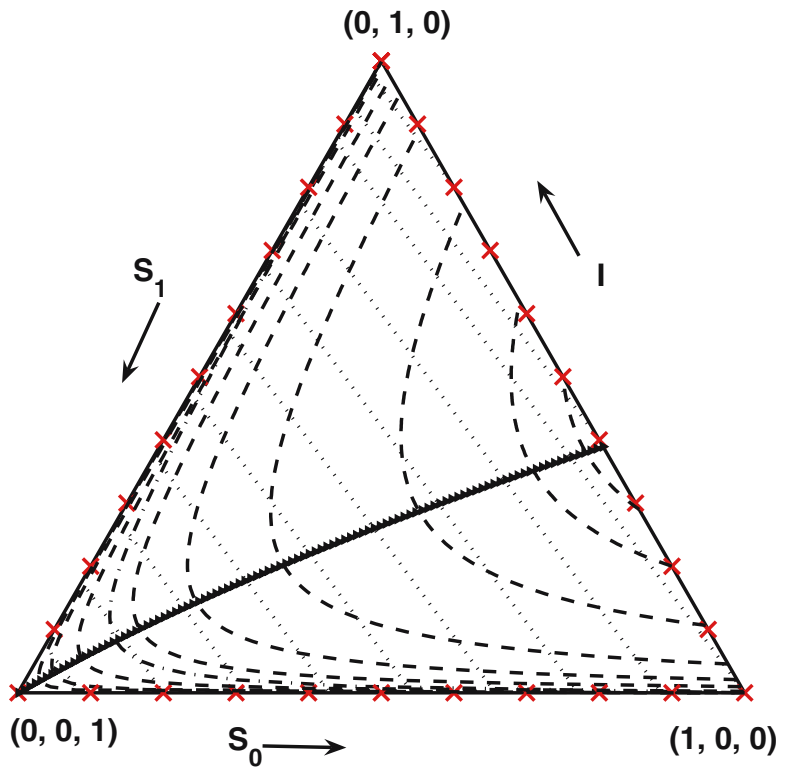

Fig. 3 This figure shows the contour lines for both the episode (straight lines) and the case (hyperbolic lines) reproduction number in a ternary plot for the parameter values $\alpha=10$ per year, $\mu=0.015$ per year, $\beta=1.8$ per year, and $r=8$. The marked line indicates the maximum case reproduction number for fixed $S_{1}$. See Sect. 4 for details

Proposition The ratio between the contact rate, $\beta$, and the critical contact rate, $\beta^{\star}$, at equilibrium is given by:

$$
\mathcal{R}=\frac{\beta}{\beta^{\star}}= \begin{cases}\frac{1}{\bar{S}_{0}+r \bar{S}_{1}} & \text { for } r \leq 1+(\mu / \alpha), \\ \frac{1}{\left(\sqrt{\frac{\bar{S}_{0}}{1-\bar{S}_{0}} \bar{I}\left(1-\frac{1}{r}\right)}+\sqrt{\left.\bar{S}_{1}\left(1+\frac{1}{r} \frac{\bar{S}_{0}}{1-\bar{S}_{0}}\right)\right)^{2}}\right.} & \text { for } r \geq 1+(\mu / \alpha),\end{cases}
$$

where the ratio $r$ is determined from the relation

$$
r=\frac{\bar{S}_{0}}{1-\bar{S}_{0}}\left(\frac{\alpha}{\mu} \frac{\bar{I}}{\overline{S_{1}}}-1\right) .
$$

Proof At equilibrium, one can find:

$$
\begin{aligned}
\mu & =\beta \bar{I} \frac{\bar{S}_{0}}{1-\bar{S}_{0}}, \\
\alpha & =\beta\left(\bar{S}_{0}+r\left(1-\bar{S}_{0}-\bar{I}\right)-\bar{I} \frac{\bar{S}_{0}}{1-\bar{S}_{0}}\right) .
\end{aligned}
$$


Substituting these expressions into the relation

$$
\beta^{\star}= \begin{cases}\alpha+\mu & \text { for } r \leq 1+(\mu / \alpha), \\ \frac{(\sqrt{\mu(r-1)}+\sqrt{\alpha})^{2}}{r} & \text { for } r \geq 1+(\mu / \alpha),\end{cases}
$$

and performing some simple calculations leads to (6). To derive (7), we find at equilibrium

$$
\begin{aligned}
\beta \bar{I} & =\frac{\mu\left(1-\bar{S}_{0}\right)}{\bar{S}_{0}}, \\
r \bar{S}_{1} & =\frac{\alpha \bar{I}-\mu \bar{S}_{1}}{\beta \bar{I}}
\end{aligned}
$$

Hence,

$$
r=\alpha \frac{\bar{I}}{\bar{S}_{1}}-\mu \frac{\bar{S}_{0}}{\mu\left(1-\bar{S}_{0}\right)}=\frac{\bar{S}_{0}}{1-\bar{S}_{0}}\left(\frac{\alpha}{\mu} \frac{\bar{I}}{\bar{S}_{1}}-1\right)
$$

Note that for the SIR $(r=0)$ and SIS $(r=1)$ models, the control effort $\mathcal{R}=R_{0}=1 / \bar{S}$ and we indeed recover the well-known identity according to which the product of the basic reproduction number and the equilibrium proportion of susceptibles equals one (where $\bar{S}$ is $\bar{S}_{0}$ or $\bar{S}_{0}+\bar{S}_{1}$, as appropriate). For other values of $r<1+(\mu / \alpha)$, the equilibrium proportion of susceptibles $\bar{S}_{0}$ and $\bar{S}_{1}$ are weighted according to their relative susceptibility $r$. For $r>1+(\mu / \alpha)$ the corresponding formula is more complicated but it can still be given in an explicit form (6).

If we know the equilibrium solution, $\left(\bar{S}_{0}, \bar{I}, \bar{S}_{1}\right)$, in addition to the model parameters $\alpha$ and $\mu$, we can evaluate $r$ from (7) and then use (6) to evaluate the effort required to eradicate the infection. The equilibrium proportions of the three epidemiological states can be estimated from a cross sectional survey. The death rate $\mu$ is obtained from demographic observations and the recovery rate $\alpha$ is estimated from the duration of the infectious period of infected cases.

The question arises whether the expression for control effort can be interpreted as a reproduction number like in the case of the SIR and SIS model. This problem will be addressed in the following sections. We can define two reproduction numbers: the episode reproduction number and the case reproduction number. The first reproduction number determines how many secondary infectious episodes are generated during one infectious episode. It depends on both $S_{0}$ and $S_{1}$. The case reproduction number asks how many new cases are generated by one case throughout life. It only depends on $S_{0}$ and on the combined duration of all infectious periods throughout the future life of a newly 
infected individual (which depends on the prevalence $I$ ). If the population is completely naive i.e. $\left(S_{0}, I, S_{1}\right)=(1,0,0)$, then both reproduction numbers are identical and equal to $R_{0}$. We will regard these numbers in a broader setting, however, where we want to express the "reproductive capacity" of an infected individual in a population with arbitrary characteristics $\left(S_{0}, I, S_{1}\right)$. One often sees the term "effective" reproduction number for such a quantity. We give two different names since the quantities defined are in general not equal. We note that, since the population composition is not fixed for their definition, all these reproduction numbers are in fact functions, in contrast to $R_{0}$. To emphasize their biological interpretation and dimension we stick, however, to the term "numbers" for all quantities.

\section{The episode reproduction number $\boldsymbol{R}_{e}$ and the case reproduction number $\boldsymbol{R}_{\boldsymbol{c}}$}

The episode reproduction number, denoted by $R_{e}$, is defined as the average number of secondary episodes (infectious periods) produced by one episode (infectious period) when the sub-populations are given by the fractions $\left(S_{0}, I, S_{1}\right)$. In the simple case of our model system, it is the product of three quantities:

$$
R_{e}=\frac{\beta\left(S_{0}+r S_{1}\right)}{(\alpha+\mu)}
$$

One can see immediately from the differential equation for $I$ in (1) that $R_{e}=1$ in steady state and that $I$ is increasing if $R_{e}>1$ and decreasing if $R_{e}<1$. Also, when the population consists of naive individuals only we see that $R_{e}=R_{0}$.

The case reproduction number, denoted by $R_{c}$, is the expected number of secondary cases produced by one infected case throughout life. It is, just like $R_{e}$, the product of three quantities: the contact rate, the proportion of individuals at risk of getting infected, and the total time of life spent in the infectious state $T(I)$. We find:

$$
R_{c}=\beta S_{0} T(I), \quad T(I)=\frac{\mu+r \beta I}{\mu(\alpha+\mu+r \beta I)} .
$$

To evaluate $T(I)$ we follow a newly infected individual $i$. This infected individual $i$ can either die with per capita death rate $\mu$ or recover without immunity to become a recovered individual with per capita recovery rate $\alpha$. Such recovered individuals can either die with per capita death rate $\mu$ or become infected again with force of infection $r \beta I$ where $I$ is the proportion of infected individuals in the whole population. We can describe the dynamics of state changes for individual $i$ by a Markov transition matrix as in [4]. For the system described above there are two states $I$ and $S_{1}$, and the transition matrix is given by

$$
G=\left(\begin{array}{cc}
-(\alpha+\mu) & r \beta I \\
\alpha & -(r \beta I+\mu)
\end{array}\right) .
$$


The expected time spent in state $1(I)$ is then given by

$$
T(I)=-\left(\begin{array}{ll}
1 & 0
\end{array}\right) G^{-1}\left(\begin{array}{l}
1 \\
0
\end{array}\right) \text {. }
$$

We now distinguish between "episode" and "case". By case we mean an individual who gets infected for the first time. It stays a case for the rest of life. In other words, we count it only once. However, concerning an episode, we count the individual during each time at which it is in the state $I$. By rearranging the equations of system (1) at steady state, one can easily see that the case reproduction number, $R_{c}$ has the following properties: $R_{c}=1$ at equilibrium (with $I$ strictly positive not necessarily at infection free equilibrium); when the population consists of naive individuals only, $R_{c}=R_{0}$.

We now study the system's dynamics in terms of the quantities defined. First, we notice that the set $S_{0}+S_{1}+I=1$ is invariant and absorbing. In order to show the isoclines of $R_{e}$ in the unit simplex we use so-called ternary plots. This graphical tool (also known as De Finetti diagram) is quite common in genetics for the representation of the relative frequencies of three genotypes [9,11]. The $S_{0}, I$, and $S_{1}$ axes are depicted with increasing values going counter clockwise in a ternary plot, and isoclines become parallel lines. Figure 3 shows the contour lines for the episode reproduction number $R_{e}$ (straight lines) and the case reproduction number $R_{c}$ (hyperbolic lines) for values of model's parameters in the area in which there are two positive endemic equilibria in addition to the IFE. Along the axis $S_{0}=0, R_{e}$ increases linearly with $S_{1}$ for $r>1$ with a maximum value equal to $r \beta / \alpha+\mu$ for $S_{1}=1$ and a minimum value equal to zero for $I=1$. The isocline which connects the two equilibria, $E^{-}$and $E^{+}$, corresponds to an $R_{e}$ value of 1 . The location of the point $E^{\star}$ in the triangle for all possible values of $r$ is shown in Fig. 4 .
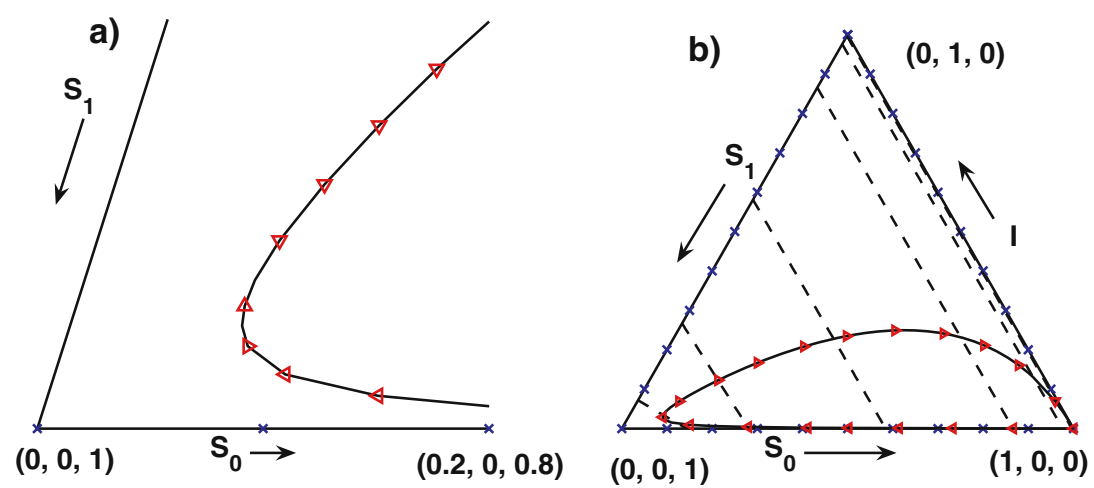

Fig. 4 This figure shows the curve in the triangle on which the point $E^{\star}$ lies for values of $r \geq 1+\mu / \alpha$ and the isoclines $R_{e}=\beta / \beta^{\star}$ for values of $r=2,20,200,2,000,20,000$, and 200,000. In Fig. 4b, we have the whole curve. At $r=1+\mu / \alpha, E^{\star}$ coincides with the IFE. When $r$ increases, the point $E^{\star}$ moves clockwise on a closed curve until reaching the IFE again when $r$ tends to infinity. An explanation to the left corner is shown in Fig. 4a. This is done for values of $r$, clockwise from the right bottom, located at head arrows $1.08,1.2,1.45,2,3.5,5.5,8.5$, and 12.5 , respectively 


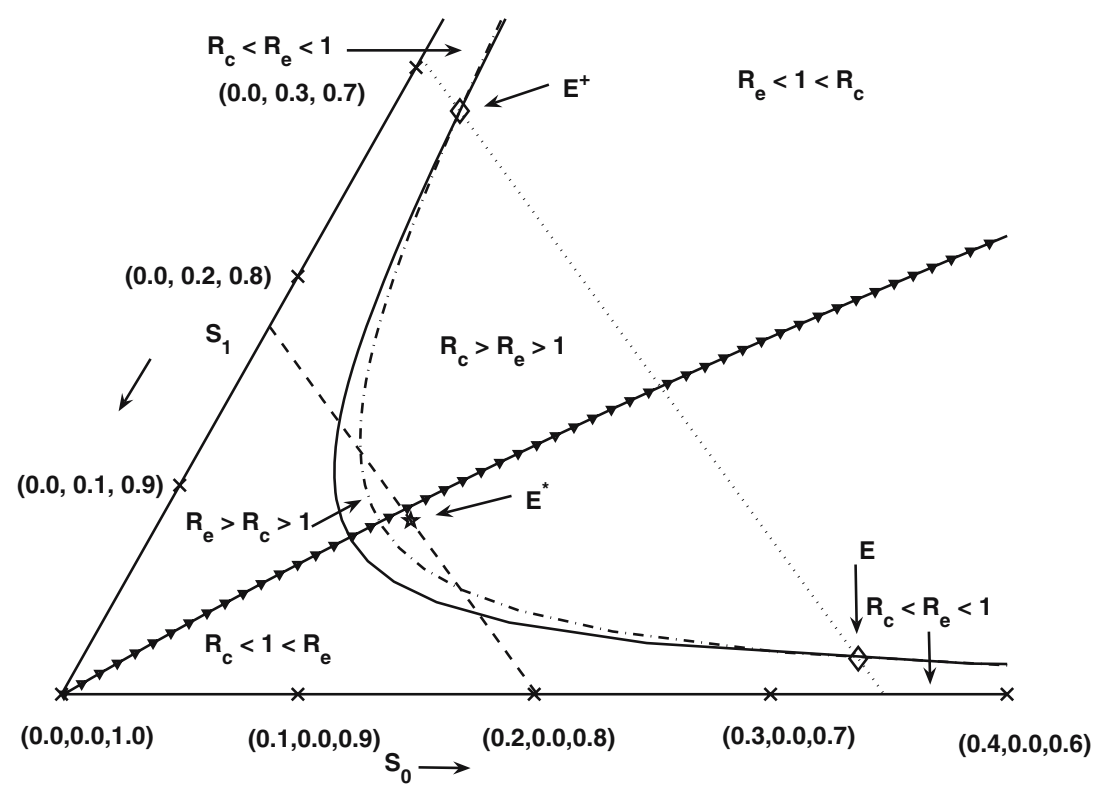

Fig. 5 Ternary plot for the episode and the case reproduction numbers in the case of backward bifurcation with two endemic equilibria $E^{+}$(stable) and $E^{-}$(unstable). The straight dotted line through these points is the contour line $R_{e}=1$. The continuous hyperbola through these points is the contour line $R_{c}=1$. The dashed dotted line is the contour line where $R_{e}=R_{c}$. The dashed line through $E^{\star}$ is the contour line $R_{e}=\beta / \beta^{\star}=\mathcal{R}$, i.e. along this line the episode reproduction number equals the minimum eradication effort. The line with arrow heads indicates the maximum $R_{c}$ for fixed $S_{1}$. The contour lines divide the triangle into areas where the inserted inequalities are valid

We are particularly interested in the isocline passing through the point $E^{\star}=$ $\left(S_{0}^{\star}, I^{\star}, S_{1}^{\star}\right)$, see Fig. 5. From formula (8) we notice that the episode reproduction number depends on the two proportions $S_{0}$ and $S_{1}$ in addition to the model parameters $\beta, r, \alpha$, and $\mu$. If we substitute these two proportions by their values at the turning point $E^{\star}$ we get $R_{e}=\mathcal{R}=\beta / \beta^{\star}$. This means that the ratio $\beta / \beta^{\star}$ equals the episode reproduction number evaluated at the turning point. Therefore, $\mathcal{R}$ is a reproduction number not only at this point but also along the isocline passing through the turning point.

\section{Transient behaviour}

Since analytical solutions of model (1) are impossible, numerical simulations have been performed. Figure 6 shows the trajectories in a ternary plot when there is a unique endemic equilibrium in addition to the IFE. If we begin the calculations at a random position within the ternary triangle, then we get the following behaviour. The initial $R_{e}$ can be less than or bigger than one. If $R_{e}>1$ we get a monotonic increase until reaching the equilibrium. This increase can be uniform in speed or it can be composed of a fast and slow component (weak growth followed by a strong one). On the other hand, if $R_{e}<1$ we get either a 
uniform decrease until reaching the equilibrium or an initial decrease followed by a sudden increase.

If we solve the system with random initial conditions in the ternary triangle with parameter values for which there are two endemic equilibria in addition to the IFE (Fig. 7), we get a combination of two different types of behaviour (one is similar to that in Fig. 6 and the other is the one in the domain of attraction of the IFE). The separatrix (broken line) divides between the domains of attraction of the IFE and the stable positive endemic equilibrium. It passes through the unstable endemic equilibrium. The isocline $R_{e}=1$ is a straight line passing through the two positive endemic states. In the domain of attraction of the IFE we notice that first $I$ and $S_{0}$ decrease, whereas $S_{1}$ increases up to a maximum value depending on the initial values until $I$ approaches zero. Susequently, $S_{1}$ decreases again and $S_{0}$ eventually increases to reach 1 . In this case, we find that the episode reproduction number $R_{e}$ is always less than one, as a consequence we get a monotonic decrease of the prevalence $I$. In the other part of the triangle we have two patterns (above and below $R_{e}=1$ ). Below the line $R_{e}=1$, both $S_{0}$ and $I$ initially increase while $S_{1}$ decreases till reaching the line $R_{e}=1$ and suddenly $S_{0}$ decreases. Above the line $R_{e}=1$, both $I$ and $S_{0}$ decrease while $S_{1}$ increases until reaching the line $R_{e}=1$ and then $I$ increases to reach the equilibrium. We see that $R_{e}$ plays an important role in the dynamics.

The important thing to notice is that for given initial prevalence $I$ we can reach both of the stable equilibria. This makes the initial conditions useless in evaluating the eradication effort in the presence of backward bifurcations. This is in contrast to the situation in the case without such bifurcations.

Fig. 6 This figure shows the ternary plot with model parameters defining a unique positive endemic equilibrium. Here, we notice that the positive stationary solution is the global attractor. The dotted straight line passing through the equilibrium point corresponds to $R_{e}=1$

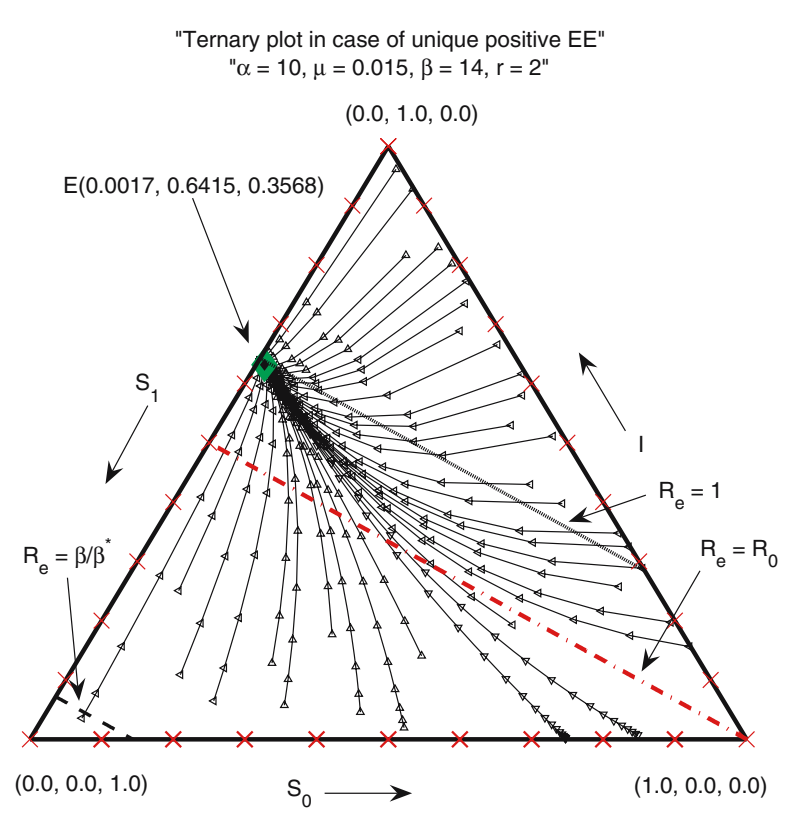


Since $\alpha$ and $\mu$ are the removal and birth/death rate, respectively, we fix them in all calculations (the length of the infectious period " $1 /(\alpha+\mu)$ " is fixed). Hence, the variability can occur with respect to both $r$ and $\beta$. We considered two different points of $(\beta, r)$ namely $(14,2)$ and $(2,7)$. They belong to two areas in the plane $\left(R_{0}, r\right)$ in Fig. 2 and are representative for the different behaviour of the solutions in the two areas (with positive endemic equilibria) of the $\left(R_{0}, r\right)$ plane.

The importance of using ternary triangles here is that we can immediately see the dynamics of the three components simultaneously and the local and global attractors. In Fig. 6 for example we notice that if we start anywhere, then there are three types of behaviour depending on the value of the episode reproduction number $R_{e}$. If $R_{e}<1$, then both $S_{0}$ and $I$ decrease while $S_{1}$ increases till reaching the global attractor. If $1<R_{e}<R_{0}$, then both $I$ and $S_{1}$ increase while $S_{0}$ decreases till reaching the global attractor. If $R_{0}<R_{e}$, then $I$ always increases while both $S_{0}$ and $S_{1}$ change to reach the equilibrium. Similarly, we can find out the different types of behaviour in Fig. 7.

\section{Discussion}

Several authors noted before that for models with backward bifurcation, the basic reproduction number can no longer be used as an indicator of the eradi-

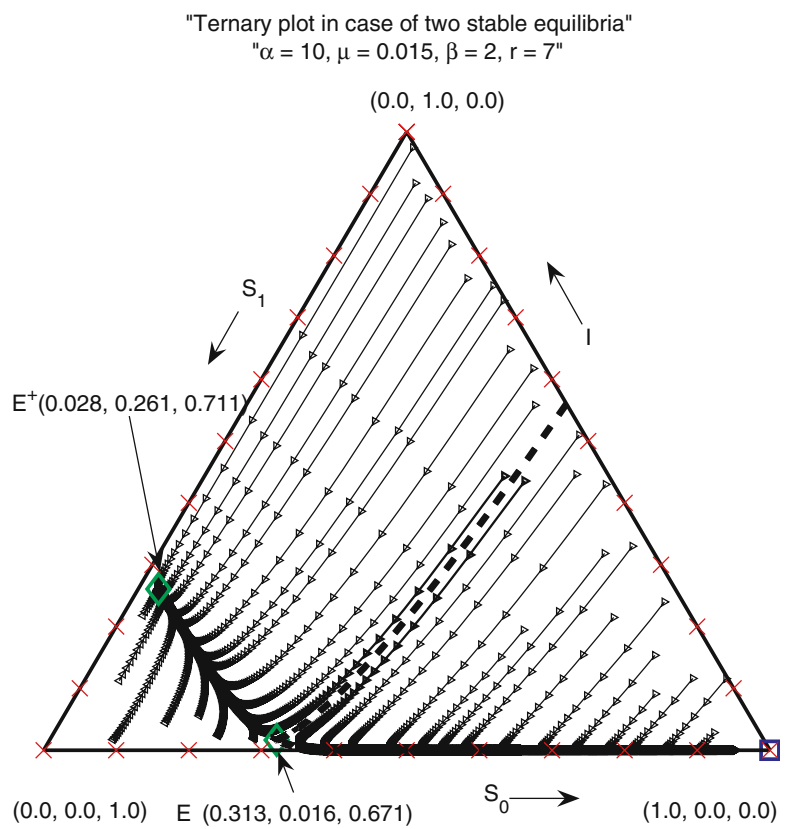

Fig. 7 This figure shows the trajectories when there are two stable equilibria $E^{+}$and the IFE with an unstable one $E^{-}$in between. The broken line indicates the separatrix which separates the domains of attraction of the two stable equilibria 
cation effort[2,6,10,12-16]. In the present paper, we provide for the first time a method to determine the eradication effort for models with backward bifurcation. It can be estimated from the composition of the population at the endemic equilibrium. We generalize the notion of the basic reproduction number for an arbitrary composition of the population in order to determine those initial conditions at which the episode reproduction number equals the eradication effort. In all models without backward bifurcation, the basic reproduction number is evaluated at the infection free equilibrium (i.e. at the IFE the episode reproduction number coincides with the basic reproduction number). At this point, $R_{0}$ represents the maximum reproduction number. In the present model with backward bifurcation the generalisation of the basic reproduction number is not unique because it is ambiguous what one should call a new case: each time someone gets infected with the agent or only the infections of naive susceptibles without infection history for that agent. In the infection free equilibrium both concepts (the episode reproduction number $R_{e}$ and the case reproduction number $R_{c}$ ) are indistinguishable from the basic reproduction number $R_{0}$. We have seen, however, that for a model with backward bifurcation the two concepts $\left(R_{e}\right.$ and $\left.R_{c}\right)$ are only identical along a hyperbolic curve. Outside this curve, they behave in opposite directions: for increasing proportion of susceptibles with previous infections the episode reproduction number increases whereas the case reproduction number decreases. It therefore does not help to take the maximum of the two reproduction numbers like in the models without backward bifurcation, because these maxima occur at opposite ends of the proportion of susceptibles with primary infections. One cannot even take the maximum of the reproduction numbers where both concepts agree with each other, because then the eradication effort would be overestimated.

We derived a formula which allows to determine the relative susceptibility $r$ in terms of the three equilibrium proportions $\bar{S}_{0}, \bar{I}$, and $\bar{S}_{1}$ (formula (7)) and an expression which involves the ratio of the life expectancy and the duration of the infectious period. If we know these four quantities we can estimate $r$ and then we have an explicit formula for the eradication effort (formula (6)) which does not explicitly involve the contact rate $\beta$. Therefore, the minimum eradication effort can be estimated from the composition of the population at equilibrium. This generalizes the simple formula that $R_{0}$ is the inverse of the proportion of susceptible at equilibrium weighted by the relative susceptibility $r$ (formula (6)).

Our method is designed for the endemic situation. One can then evaluate the minimum eradication effort $\mathcal{R}$ completely in terms of the equilibrium proportions of the three components of the population. If $\mathcal{R}<1$ the infection dies out, if $\mathcal{R}>1$ the infection can persist. Therefore, we have a clear concept that provides insight into the eradication effort needed. For the epidemic situation, however, the matter is more complicated and less clear cut. Suppose the infectious agent is introduced into a naive population, with $R_{0}>1$ and the relative susceptibility $r>1+\mu / \alpha$. If we can achieve $\mathcal{R}<1$ quickly (i.e. if we can reduce the contact rate $\beta$ to a value less than $\beta^{\star}$, i.e. $R_{0}$ to a value less than $R_{0}^{\star}$, quickly) then the infection can die out because we are still in the domain of 
attraction of the IFE (infection-free-equilibrium). In that case $\mathcal{R}$ gives the control effort needed. When this cannot be achieved quickly or when $R_{0}$ reduces to a value less than one but greater than $R_{0}^{\star}$, then there are two possibilities (see Fig. 7): either the initial episode reproduction number is bigger than one and hence the infection persists, or the initial episode reproduction number is less than one. For the latter, the infection dies out if we start in the domain of attraction of the IFE (i.e. on the right of the separatrix) or it persists if we start in the domain of attraction of the positive stable endemic equilibrium (i.e. on the left of the separatrix). Hence, the initial episode reproduction number does not help in the elimination process.

Application of our method to models like those in [7,8,10,15] and [2] will be published elsewhere. See [17] for the application to models like those in [7,15].

\section{References}

1. Brauer, F.: Backward bifurcations in simple vaccination models. J. Math. Anal. Appl. 298, 418-431 (2004)

2. van Boven, M., Mooi, F.R., Schellekens, J.F.P., de Melker, H.E., Kretzschmar, M.: Pathogen adaptation under imperfect vaccination: implications for pertussis. Proc. R. Soc. Lond. B 272, 1617-1624 (2005)

3. Castillo-Chavez, C., Song, B.: Dynamical models of tuberculosis and their applications. Math. Biosci. Eng. 1, 361-404 (2004)

4. Diekmann, O., Heesterbeek, J.A.P.: Mathematical Epidemiology of Infectious Diseases: Model Building, Analysis and Interpretation. Wiley, Chichester (2000)

5. Diekmann, O., Heesterbeek, J.A.P., Metz, J.A.J.: On the definition and the computation of the basic reproduction ratio $R_{0}$ in models for infectious diseases in heterogeneous populations. J. Math. Biol. 28, 365-382 (1990)

6. van den Driessche, P., Watmough, J.: A simple SIS epidemic model with a backward bifurcation. J. Math. Biol. 40, 525-540 (2000)

7. Hadeler, K.P., Castillo-Chavez, C.: A core group model for disease transmission. Math. Biosci. 128, 41-55 (1995)

8. Hadeler, KP., van den Driessche, P.: Backward bifurcation in epidemic control. Math. Biosci. 146, 15-35 (1997)

9. Hadeler, K.P., Liberman, U.: Selection models with fertility differences. J. Math. Biol. 2, 19-32 (1975)

10. Greenhalgh, D., Diekmann, O., de Jong, M.C.M.: Subcritical endemic steady states in mathematical models for animal infections with incomplete immunity. Math. Biosci. 165, 1-25 (2000)

11. Ineichen, R., Batschelet, E.: Genetic selection and de Finetti diagrams. J. Math. Biol. 2, 33-39 (1975)

12. Kribs-Zaleta, C.M.: Center manifold and normal forms in epidemic models. In: C. CastilloChavez et al. (eds.) Mathematical Approaches for Emerging and Reemerging Infectious Diseases: Models, Methods and Theory, IMA 125, Springer pp. 269-286, New York (2002)

13. Kribs-Zaleta, C.M., Martcheva, M.: Vaccination strategies and backward bifurcation in an age-since-infection structured model. Math. Biosci. 177, 178, 317-332 (2002)

14. Kribs-Zaleta, C.M., Velasco-Hernandez, J.X.: A simple vaccination model with multiple endemic states. Math. Biosci. 164, 183-201 (2000)

15. Moghadas, S.M.: Analysis of an epidemic model with bistable equilibria using the Poincaré index. Appl. Math. Comput. 149, 689-702 (2004)

16. Moghadas, S.M.: Modelling the effect of imperfect vaccines on disease epidemiology. Discret Contin Dyn Syst-Ser B4, 999-1012 (2004)

17. Safan, M.: Spread of infectious diseases: impact on demography, and the eradication effort in models with backward bifurcation. Dissertation, University of Tuebingen, Germany (2006)

18. Seydel, R.: From Equilibrium to Chaos: Practical Bifurcation and Stability Analysis. Elsvier, Amesterdam (1988) 